\title{
Freier Zugang zu Fachliteratur - Cochrane Library
}

\section{Stefanie Hostettler}

Dr. sc. ETH Zürich, Abteilung Daten, Demographie und Qualität (DDQ) der FMH

\section{Cochrane schafft verlässliche Gesund- heitsinformationen}

Tagtäglich entstehen neue Informationen in der klinischen Forschung. Als Einzelner eine wissenschaftlich fundierte Antwort zu einer medizinischen Fragestellung zu erhalten wird zunehmend komplexer. Dabei besteht auch die Gefahr, diese komplexen Inhalte falsch zu interpretieren. Das weltweite Netzwerk Cochrane bietet hier Unterstützung. Es erstellt seit über 25 Jahren systematische Übersichtsarbeiten, in denen Forschungsergebnisse zu definierten Fragestellungen zu Therapie, Prävention und Diagnose zusammengefasst werden. Die Autorinnen und Autoren arbeiten in über 50 Review-Gruppen zusammen, die sich um jeweils einen Themenkreis, wie zum Beispiel muskuloskelettale Erkrankungen, Schwangerschaft und Geburtshilfe oder Schmerz- und Palliativmedizin, kümmern. Dem Netzwerk sind auch Patientengruppen angeschlossen. Durch internationale Koordination wer-

\section{Zusammenfassung}

Cochrane erstellt systematische Übersichtsarbeiten zu medizinischen Forschungsfragen und schafft damit eine zuverlässige Grundlage für die evidenzbasierte Medizin. Seit 2016 haben alle in der Schweiz wohnhaften Personen kostenlosen Zugang zur Cochrane Library. Aktuell wird der Zugang zur Cochrane Library von der Akademie der Medizinischen Wissenschaften (SAMW), dem Bundesamt für Gesundheit und den medizinischen Universitätsbibliotheken finanziert. Neu ab 2020 auch von der FMH. Es gibt viele auch innerhalb der Ärzteschaft -, die den freien Zugang zur Cochrane Library nicht kennen oder nutzen. Aus diesem Grund wird die Cochrane Library in einer vierteiligen Artikelserie in den nächsten Ausgaben der Schweizerischen Ärztezeitung vorgestellt. Dieser erste Artikel enthält allgemeine Informationen zur evidenzbasierten Medizin und zu Cochrane. In den nachfolgenden, praxisorientierten Artikeln berichten ein Hausarzt und ein Spezialmediziner über ihre Erfahrung im Umgang mit der Cochrane Library. Im letzten Artikel erscheint ein Interview mit Dr. med. Erik von Elm, Direktor von Cochrane Schweiz. den thematische Überlappungen vermieden, so dass es zu einer Fragestellung nur jeweils einen Cochrane Review gibt.

Cochrane Schweiz setzt sich dafür ein, dass Evidenz dorthin gelangt, wo sie angewendet und benötigt wird (z.B. Praktiker, Patienten, Entscheidungsträger).

\section{Cochrane fördert die Qualität in der Medizin}

Das Ziel von Cochrane ist es, mit leicht zugänglicher und vertrauenswürdiger wissenschaftlicher Evidenz eine Grundlage für gute Entscheidungen zu schaffen. Ob Ärztinnen und Ärzten, Pflegefachpersonen, Patientinnen und Patienten, Wissenschaftlerinnen und Wissenschaftlern oder Forschungsförderern, Cochrane steht jedem offen, der für gesundheitsbezogene Entscheidungen hochwertige Informationen verwenden möchte.

Für Interessierte ohne fachlichen Hintergrund gibt es «Cochrane Kompakt» mit aktuell über 1900 laienverständlichen Zusammenfassungen der Cochrane Reviews in deutscher Sprache. In französischer Sprache stehen aktuell sogar über 5500 dieser Zusammenfassungen bereit. Im Cochrane Blog "Wissen was wirkt» werden Gesundheitsthemen diskutiert wie z.B. "Gedächtnisstörung nach einem Schlaganfall: hilft die kognitive Rehabilitation?», "Vorbeugung von Diabetes Typ 2 - was wirklich hilft», «Honig gegen Husten: wirkt Grossmutters Hausmittel doch?».

Seit 2015 wurden über 200 Blog-Beiträge veröffentlicht. Die Website «Wissen was wirkt» wurde 2019 bereits über $14000 \mathrm{Mal}$ pro Monat aufgerufen. Nicht zuletzt kommt den Cochrane Reviews eine wichtige Bedeutung als evidenzbasierte Grundlage für politische Entscheide im Gesundheitswesen zu. So auch bei der Herausgabe von Empfehlungen (z.B. Impfempfehlungen), bei der Finanzierung von Versorgungsleistungen (z.B. Brustkrebsscreening) oder für Health Technology Assessments (systematische Bewertung medizinischer Verfahren und Technologien). 


\section{Eine Nationallizenz für den freien Zugang - mitgetragen von der FMH}

Der Zugang zu wissenschaftlicher Literatur inkl. der Cochrane Library ist häufig auf akademische Institutionen beschränkt. Auf Initiative der SAMW haben alle in der Schweiz wohnhaften Personen seit 2016 kostenlosen Zugang zur Cochrane Library. Schon in den ersten Wochen haben sich die Nutzerzahlen verdoppelt; aktuell werden in der Schweiz pro Jahr durchschnittlich 163000 Reviews im Volltext heruntergeladen. Die Nationallizenz wird von 2016 bis 2020 von der SAMW, dem Bundesamt für Gesundheit und den medizinischen Universitätsbibliotheken finanziert. Ab 2020 wird sich die FMH ebenfalls an den Kosten der Nationallizenz beteiligen

Der FMH ist es ein Anliegen, evidenzbasierte Medizin zu fördern und das Angebot des freien Zugangs zur Cochrane Library bekannter zu machen.

\section{Eine vierteilige Artikelserie zur Cochrane Library}

Um das Angebot der Cochrane Library bekannter zu machen, wird in dieser und den nächsten drei Ausgaben der Schweizerischen Ärztezeitung der Nutzen der Cochrane Reviews aus verschiedenen Perspektiven aufgezeigt. In der nächsten Ausgabe berichtet Dr. med. Bruno Kissling von seinen Erfahrungen, wie Evidenz von Cochrane in der Hausarztmedizin genutzt werden kann. Im darauf folgenden Artikel wird Prof. Dr. med. Fabian Krause aus der Sicht eines orthopädischen Chirurgen Cochrane beleuchten. Im letzten Artikel gibt der Direktor von Cochrane Schweiz, Dr. med. Erik von Elm, in einem Interview Auskunft zum Angebot von Cochrane und nimmt Bezug zu den Inhalten der vorangehenden Artikel.
FMH/SAQM,

Elfenstrasse 18 CH-3000 Bern 15 Tel. 031359111 ddq[at]fmh.ch

\section{Über Cochrane}

Cochrane ist ein globales, unabhängiges Netzwerk von Wissenschaftlerinnen und Wissenschaftlern, Gesundheitsfachleuten, Patienten und Patientinnen sowie anderen Personen mit gesundheitsbezogenen Interessen. Zur Arbeit von Cochrane tragen ca. 11000 Mitglieder und über 68000 Unterstützer aus über 130 Ländern bei. Auf wissenschaftliche Methoden gestützt, stellen sie Gesundheitsinformationen bereit, die zuverlässig und frei von kommerziellen Sponsorengeldern sind. Jeder Cochrane Review widmet sich einer klar formulierten Fragestellung. $\mathrm{Zu}$ deren Beantwortung sucht ein Autorenteam alle vorhandenen Originalstudien, welche die zuvor definierten Einschlusskriterien erfüllen. Anschliessend werden die eingeschlossenen Studien bewertet, um zu bestimmen, ob es zuverlässige Evidenz zu einer bestimmten Behandlung, Diagnostik oder vorbeugenden Massnahme gibt. Wenn möglich, werden die Einzelergebnisse in einer Metaanalyse kombiniert. Cochrane Reviews werden vor der Veröffentlichung im Peer-Review-Verfahren von Fachexperten begutachtet. Über 8000 Reviews sind bisher auf www.cochranelibrary. com zugänglich. Neben dem oft sehr ausführlichen Volltext stehen verschiedene Kurzformate zur Verfügung. Für die klinische Praxis besonders relevante Reviews werden unter "Clinica Answers» in einem Frage-Antwort-Format dargestellt.

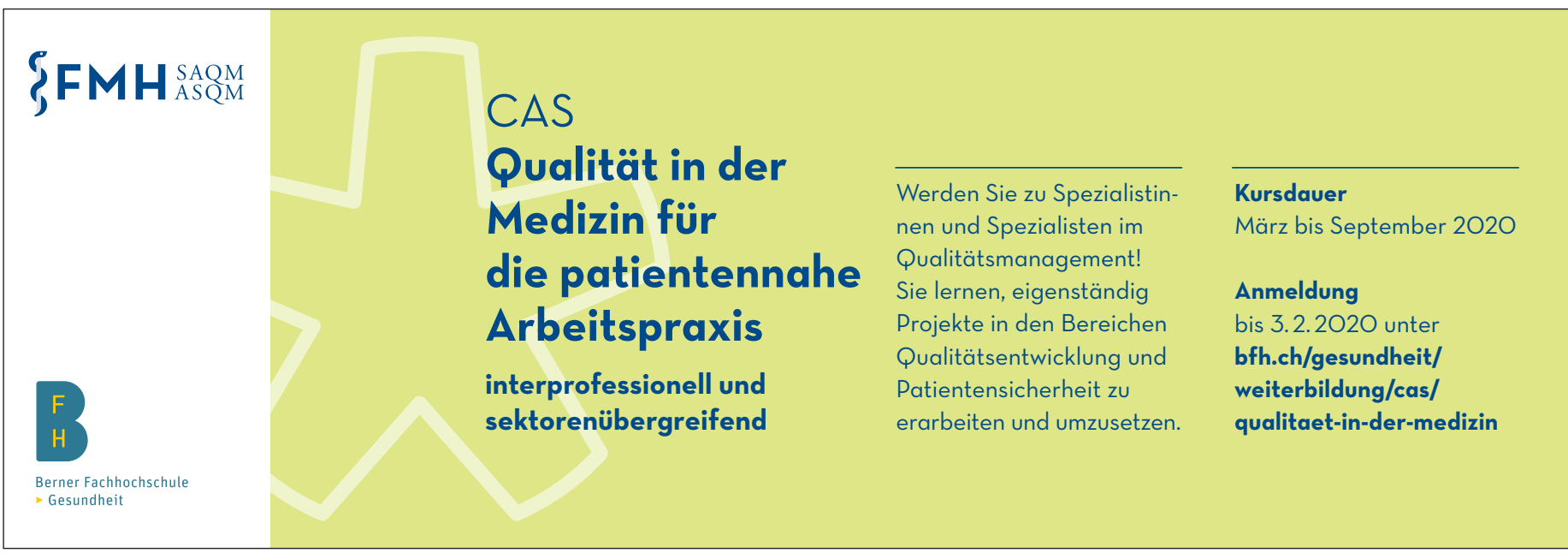

